\title{
Stability and connectivity of real foodwebs predicted by mass constraints on maximum consumption and density dependence
}

\author{
Tianyun Lin ${ }^{1}$, Daniel Wieczynski ${ }^{2}$, and Van Savage ${ }^{1}$ \\ ${ }^{1} \mathrm{UCLA}$ \\ ${ }^{2}$ Duke University
}

October 1, 2020

\begin{abstract}
Understanding which foodwebs thrive or collapse is a major challenge that has been mostly studied in terms of topology and interaction strength. Yet the relative importance of these properties is hotly debated due to limited research on how they interact and which forces generate them. Here, we construct a foodweb model that incorporates mass-based constraints on density dependence, maximum consumption rate, and the likelihood and strength of interactions, which in turn control overall topologies and interaction strength distributions. Our model predicts both stability and connectivity that closely match real foodwebs ranging widely in size (29-163 species) and connectivity (113-1086 interactions). Despite their absence in most research, density dependence and maximum consumption rate are not only required to accurately predict stability but have stronger impacts than the more-frequently-studied interaction strength. Our results demonstrate that predicting foodweb stability requires simultaneously considering multiple foodweb properties - all of which naturally emerge from species masses.
\end{abstract}

\section{Hosted file}

size_stability_manuscript_final.pdf available at https://authorea.com/users/363525/articles/ 484261-stability-and-connectivity-of-real-foodwebs-predicted-by-mass-constraints-onmaximum-consumption-and-density-dependence 